\title{
RETIRED A STARS AND THEIR COMPANIONS. VI. A PAIR OF INTERACTING EXOPLANET PAIRS AROUND THE SUBGIANTS 24 SEXTANIS AND HD 200964*
}

\author{
John Asher Johnson ${ }^{1}$, Matthew Payne ${ }^{2}$, Andrew W. Howard ${ }^{3}$, Kelsey I. Clubb ${ }^{4}$, Eric B. Ford ${ }^{2}$, Brendan P. Bowler $^{5}$, \\ Gregory W. Henry ${ }^{6}$, Debra A. Fischer ${ }^{7}$, Geoffrey W. Marcy ${ }^{3}$, John M. Brewer ${ }^{7}$, Christian Schwab ${ }^{7,8}$, Sabine RefFert $^{8}$, \\ AND Thomas B. Lowe ${ }^{9}$ \\ ${ }^{1}$ California Institute of Technology, Department of Astrophysics, MC 249-17, Pasadena, CA 91125, USA; johnjohn@ astro.caltech.edu \\ ${ }^{2}$ Department of Astronomy, University of Florida, 211 Bryant Space Science Center, P.O. Box 112055, Gainesville, FL 32611-2055, USA \\ ${ }^{3}$ Department of Astronomy, University of California, Mail Code 3411, Berkeley, CA 94720, USA \\ ${ }^{4}$ Department of Physics \& Astronomy, San Francisco State University, San Francisco, CA 94132, USA \\ ${ }^{5}$ Institute for Astronomy, University of Hawai'i, Honolulu, HI 96822, USA \\ ${ }^{6}$ Center of Excellence in Information Systems, Tennessee State University, 3500 John A. Merritt Boulevard, Box 9501, Nashville, TN 37209, USA \\ ${ }^{7}$ Department of Astronomy, Yale University, New Haven, CT 06511, USA \\ ${ }^{8}$ ZAH-Landessternwarte, Königstuhl 12, D-69117 Heidelberg, Germany \\ ${ }^{9} \mathrm{UCO} /$ Lick Observatory, Santa Cruz, CA 95064, USA \\ Received 2010 June 7; accepted 2010 October 25; published 2010 December 10
}

\begin{abstract}
We report radial velocity (RV) measurements of the G-type subgiants 24 Sextanis (= HD 90043) and HD 200964. Both are massive, evolved stars that exhibit periodic variations due to the presence of a pair of Jovian planets. Photometric monitoring with the T12 $0.80 \mathrm{~m}$ APT at Fairborn Observatory demonstrates both stars to be constant in brightness to $\leqslant 0.002 \mathrm{mag}$, thus strengthening the planetary interpretation of the RV variations. Based on our dynamical analysis of the RV time series, $24 \mathrm{Sex}$ b, c have orbital periods of 452.8 days and 883.0 days, corresponding to semimajor axes $1.333 \mathrm{AU}$ and $2.08 \mathrm{AU}$, and minimum masses $1.99 M_{\mathrm{Jup}}$ and $0.86 M_{\mathrm{Jup}}$, assuming a stellar mass $M_{\star}=1.54 M_{\odot}$. HD $200964 \mathrm{~b}$, c have orbital periods of 613.8 days and 825.0 days, corresponding to semimajor axes $1.601 \mathrm{AU}$ and $1.95 \mathrm{AU}$, and minimum masses $1.99 M_{\text {Jup }}$ and $0.90 M_{\text {Jup }}$, assuming $M_{\star}=1.44 M_{\odot}$. We also carry out dynamical simulations to properly account for gravitational interactions between the planets. Most, if not all, of the dynamically stable solutions include crossing orbits, suggesting that each system is locked in a mean-motion resonance that prevents close encounters and provides long-term stability. The planets in the 24 Sex system likely have a period ratio near 2:1, while the HD 200964 system is even more tightly packed with a period ratio close to 4:3. However, we caution that further RV observations and more detailed dynamical modeling will be required to provide definitive and unique orbital solutions for both cases, and to determine whether the two systems are truly resonant.
\end{abstract}

Key words: planets and satellites: detection - planets and satellites: formation - planets and satellites: fundamental parameters - planets and satellites: individual (HD200964 b, HD200964 c, 24 Sex b, 24 Sex c) - stars: individual (24 Sex, HD200964) - techniques: radial velocities

\section{INTRODUCTION}

The giant planets thus far discovered around other stars exhibit a wide variety of orbital characteristics that are very different from the properties of the planets in our solar system. For example, exoplanets rarely reside in circular orbits and many of them have semimajor axes 1-2 orders of magnitude smaller than those of Jupiter and Saturn. However, at least one characteristic of exoplanets appears to be shared with the constituents of the solar system: planets often come in bunches. As Wright et al. (2009) recently showed, 28\% of apparently singleton exoplanets are later discovered to reside in systems containing two or more components. As the precision and time baselines of Doppler surveys increase, and as more planets are discovered from wide-field transit surveys (Bakos et al. 2009; Cameron et al. 2007), direct imaging (Marois et al. 2008; Kalas et al. 2008), and microlensing (Gaudi et al. 2008; Gould et al. 2010), the currently measured multiplicity rate will likely prove to be a lower bound.

\footnotetext{
* Based on observations obtained at the Lick Observatory, which is operated by the University of California, and W. M. Keck Observatory, which is operated jointly by the University of California and the California Institute of Technology.
}

Multiplanet systems are manifested in radial velocity (RV) time series as either a single-planet Keplerian motion superimposed atop a partial longer-period orbit, or as multiple, timeresolved orbits (e.g., Wright et al. 2009). Those in the former category will gradually come into focus as the time baselines of Doppler surveys lengthen, and these "trend" systems are becoming recognized as promising direct-imaging targets. The latter category, with their well-characterized orbits, is extremely valuable for understanding the origins of planets and the evolution of their orbital architectures (Ford 2006b).

Observed deviations from pure Keplerian motions reveal gravitational interactions among planets that serve as fossil records of past close encounters and/or convergent orbital migration (Malhotra 2002; Wu \& Goldreich 2002). A prime example is the system of planets orbiting $v$ Andromedae (Butler et al. 1999). Ford et al. (2005) demonstrated through dynamical modeling that the current orbital configuration shows evidence of a violent planet-planet scattering event in the distant past. Similarly, the two Jovian planets in the Gl 876 system currently reside in a mean-motion resonance (MMR) that may be a reflection of differential migration after the planets' formation (Marcy et al. 2001; Lee 2004; Laughlin et al. 2005). Thus, the discovery of exoplanets in MMRs is strong support for the inward orbital migration that is often invoked to explain the 
common existence of giant planets well within the canonical "snow line."

Additionally, gravitational interactions among resonant planets can also place constraints on both the system inclination with respect to the sky and mutual inclinations between the planets, and thereby remove the $\sin i$ ambiguity and provide absolute measurements of the planet masses (Rivera et al. 2005; Correia et al. 2010). Interactions observed in certain types of multiplanet systems can reveal the interior structures of gas giant planets in vivid detail. In the dramatic case of the system of planets around HAT-P-13, the inner planet transits its host star and experiences additional gravitational perturbations from an outer planet near 1 AU (Bakos et al. 2009; Winn et al. 2010). Depending on the inclinations of the planets in the system, precise follow-up measurements may provide estimates of the tidal Love number and $Q$ value of the inner planet to a higher precision than is possible for Jupiter (Batygin et al. 2009; Mardling 2010).

We are conducting a Doppler survey of intermediate-mass subgiant stars at the Lick and Keck Observatories with the goal of understanding the influence of stellar mass on the physical properties, orbital architectures, and multiplicity rates of planetary systems. Our survey has resulted in the discovery of 14 new singleton exoplanets (Johnson et al. 2006b, 2007, 2008, 2010; Peek et al. 2009; Bowler et al. 2010). In this contribution, we announce the discovery of two pairs of Jovian planets orbiting the subgiants 24 Sextanis (= HD 90043) and HD 200964.

\section{SPECTROSCOPIC OBSERVATIONS}

We began observations of 24 Sex and HD 200964 at Lick Observatory in 2004-2005 as part of our Doppler survey of intermediate-mass subgiants. Details of the survey, including target selection and observing strategy, are given in Johnson et al. (2006a), Peek et al. (2009), and Bowler et al. (2010). In 2007, we expanded our survey of subgiants at Keck Observatory (Johnson et al. 2010) and we added 24 Sex and HD 200964 to our Keck target list for additional monitoring.

At Lick Observatory, the Shane $3 \mathrm{~m}$ and $0.6 \mathrm{~m}$ Coude Auxiliary Telescopes (CAT) feed the Hamilton spectrometer (Vogt 1987), and observations at Keck Observatory were obtained using the High Resolution Echelle Spectrometer (HIRES; Vogt et al. 1994). Doppler shifts are measured from each observation using the iodine cell method described by Butler et al. (1996, see also Marcy \& Butler 1992). A temperature-controlled Pyrex cell containing gaseous iodine is placed at the entrance slit of the spectrometer. The dense set of narrow molecular lines imprinted on each stellar spectrum from 5000 to $6000 \AA$ provides a robust wavelength scale for each observation, as well as information about the shape of the spectrometer's instrumental response (Valenti et al. 1995).

At Lick, typical exposure times of 60 minutes on the CAT and 5 minutes on the $3 \mathrm{~m}$ yield a signal-to-noise ratio $(\mathrm{S} / \mathrm{N})$ of $\approx 120$ at the center of the iodine region $(\lambda=5500 \AA)$, providing a velocity precision of $4.0-5.0 \mathrm{~m} \mathrm{~s}^{-1}$. At Keck, typical spectra have $\mathrm{S} / \mathrm{N} \approx 180$ at $5500 \AA$, resulting in a velocity precision of $1.5-2.0 \mathrm{~m} \mathrm{~s}^{-1}$

In addition to the internal, photon-limited uncertainties, the RV measurements include an additional noise term due to stellar "jitter" - velocity noise in excess of internal errors due to astrophysical sources such as pulsation and rotational modulation of surface features (Saar et al. 1998; Wright 2005). We therefore adopt a jitter value of $5 \mathrm{~m} \mathrm{~s}^{-1}$ for our subgiants based on the estimate of Johnson et al. (2010). This jitter term
Table 1

Stellar Parameters

\begin{tabular}{lcc}
\hline \hline Parameter & $24 \mathrm{Sex}^{\mathrm{a}}$ & HD 200964 \\
\hline$V$ & $6.61(0.04)$ & $6.64(0.04)$ \\
$M_{V}$ & $2.17(0.06)$ & $2.35(0.07)$ \\
$B-V$ & $0.92(0.01)$ & $0.880(0.009)$ \\
Distance $(\mathrm{pc})$ & $74.8(4.9)$ & $68.4(4.8)$ \\
{$[\mathrm{Fe} / \mathrm{H}]$} & $-0.03(0.04)$ & $-0.15(0.04)$ \\
$T_{\mathrm{eff}}(\mathrm{K})$ & $5098(44)$ & $5164(44)$ \\
$V_{\text {rot }} \sin i\left(\mathrm{~km} \mathrm{~s}^{-1}\right)$ & $2.77(0.5)$ & $2.28(0.5)$ \\
$\log g$ & $3.5(0.1)$ & $3.6(0.1)$ \\
$M_{*}\left(M_{\odot}\right)$ & $1.54(0.08)$ & $1.44(0.09)$ \\
$R_{*}\left(R_{\odot}\right)$ & $4.9(0.08)$ & $4.3(0.09)$ \\
$L_{*}\left(L_{\odot}\right)$ & $14.6(0.1)$ & $11.6(0.4)$ \\
Age $(\mathrm{Gyr})$ & $2.7(0.4)$ & $3.0(0.6)$ \\
$\log R_{H K}^{\prime}$ & -5.1 & -5.1 \\
\hline
\end{tabular}

Note. ${ }^{a}$ HD 90043.

is added in quadrature to the internal errors before determining the Keplerian orbital solutions. For the dynamical analysis in Section 6 we allow the jitter to vary as a free parameter in the fitting process.

\section{STELLAR PROPERTIES}

Atmospheric parameters of the target stars are estimated from iodine-free, "template" spectra using the LTE spectroscopic analysis package Spectroscopy Made Easy (SME; Valenti \& Piskunov 1996), as described by Valenti \& Fischer (2005) and Fischer \& Valenti (2005). To constrain the low surface gravities of the evolved stars we used the iterative scheme of Valenti et al. (2009), which ties the SME-derived value of $\log g$ to the gravity inferred from the Yonsei-Yale ( $\mathrm{Y}^{2}$; Yi et al. 2004) stellar model grids. The analysis yields a best-fitting estimate of $T_{\text {eff }}, \log g,[\mathrm{Fe} / \mathrm{H}]$, and $V_{\text {rot }} \sin i$. The properties of our targets from Lick and Keck are listed in the fourth edition of the Spectroscopic Properties of Cool Stars Catalog (SPOCS IV; J. A. Johnson et al. 2011, in preparation). We adopt the SME parameter uncertainties described in the error analysis of Valenti \& Fischer (2005).

The luminosity of each star is estimated from the apparent $V$-band magnitude and the parallax from Hipparcos (van Leeuwen 2007), together with the bolometric correction from VandenBerg \& Clem (2003). From $T_{\text {eff }}$ and luminosity, we determine the stellar mass, radius, and an age estimate by associating those observed properties with a model from the $\mathrm{Y}^{2}$ stellar interior calculations (Yi et al. 2004). We also measure the chromospheric emission in the Ca II H\&K line cores (Wright et al. 2004), providing an $S$ value on the Mount Wilson system, which we convert to $R_{H K}^{\prime}$ as per Noyes et al. (1984). The stellar properties of the host stars are summarized in Table 1.

\section{KEPLERIAN ORBITAL SOLUTIONS}

In this section, we present the RV time series for both stars and the initial orbital analysis, which consists of the sum of two Keplerians without gravitational interaction. In Section 6, we present the results of our Newtonian dynamical analysis for each system, which properly accounts for non-Keplerian motion due to gravitational interactions between the planets and host star.

To search for the best-fitting, two-planet orbital solution for each time series we use the partially linearized technique presented by Wright \& Howard (2009), as implemented in 
Table 2

Radial Velocities for 24 Sextanis

\begin{tabular}{|c|c|c|c|}
\hline $\begin{array}{l}\text { JD } \\
-2440000\end{array}$ & $\begin{array}{c}\mathrm{RV} \\
\left(\mathrm{m} \mathrm{s}^{-1}\right)\end{array}$ & $\begin{array}{l}\text { Uncertainty } \\
\left(\mathrm{m} \mathrm{s}^{-1}\right)\end{array}$ & Telescope \\
\hline 13405.936 & -34.98 & 4.24 & $\mathrm{~L}$ \\
\hline 13435.836 & -37.40 & 4.05 & $\mathrm{~L}$ \\
\hline 13502.774 & 3.04 & 3.76 & $\mathrm{~L}$ \\
\hline 13517.768 & 8.07 & 3.90 & $\mathrm{~L}$ \\
\hline 13719.011 & -13.68 & 3.46 & $\mathrm{~L}$ \\
\hline 14131.051 & 38.40 & 4.17 & $\mathrm{~L}$ \\
\hline 14133.931 & 37.92 & 3.49 & $\mathrm{~L}$ \\
\hline 14146.787 & 28.17 & 4.15 & $\mathrm{~L}$ \\
\hline 14150.947 & 34.70 & 6.03 & $\mathrm{~L}$ \\
\hline 14168.823 & 28.25 & 4.33 & $\mathrm{~L}$ \\
\hline 14169.860 & 30.86 & 3.65 & $\mathrm{~L}$ \\
\hline 14178.699 & 36.12 & 4.10 & $\mathrm{~L}$ \\
\hline 14216.839 & -6.66 & 4.22 & $\mathrm{~L}$ \\
\hline 14218.709 & -8.74 & 6.44 & $\mathrm{~L}$ \\
\hline 14232.713 & -4.29 & 4.05 & $\mathrm{~L}$ \\
\hline 14254.688 & -24.93 & 6.01 & $\mathrm{~L}$ \\
\hline 14255.689 & -33.28 & 4.29 & $\mathrm{~L}$ \\
\hline 14266.699 & -25.63 & 4.77 & $\mathrm{~L}$ \\
\hline 14269.695 & -28.99 & 5.87 & $\mathrm{~L}$ \\
\hline 14274.719 & -31.09 & 4.86 & $\mathrm{~L}$ \\
\hline 14378.045 & -24.52 & 6.79 & $\mathrm{~L}$ \\
\hline 14402.054 & 2.53 & 5.26 & $\mathrm{~L}$ \\
\hline 14433.046 & 24.41 & 5.29 & $\mathrm{~L}$ \\
\hline 14445.938 & 4.46 & 3.08 & $\mathrm{~L}$ \\
\hline 14458.050 & 4.53 & 4.31 & $\mathrm{~L}$ \\
\hline 14482.959 & 11.65 & 3.89 & $\mathrm{~L}$ \\
\hline 14504.855 & 8.92 & 3.36 & $\mathrm{~L}$ \\
\hline 14525.946 & 25.65 & 4.37 & $\mathrm{~L}$ \\
\hline 14548.900 & 22.38 & 3.29 & $\mathrm{~L}$ \\
\hline 14572.872 & 13.31 & 4.54 & $\mathrm{~L}$ \\
\hline 14573.724 & 16.22 & 3.77 & $\mathrm{~L}$ \\
\hline 14617.764 & 11.34 & 5.29 & $\mathrm{~L}$ \\
\hline 14620.705 & -1.69 & 3.78 & $\mathrm{~L}$ \\
\hline 14621.716 & 11.26 & 5.22 & $\mathrm{~L}$ \\
\hline 14627.708 & 11.39 & 3.87 & $\mathrm{~L}$ \\
\hline 14650.692 & -2.99 & 6.79 & $\mathrm{~L}$ \\
\hline 14762.024 & -24.44 & 7.00 & $\mathrm{~L}$ \\
\hline 14786.078 & -13.05 & 6.03 & $\mathrm{~L}$ \\
\hline 14806.150 & -22.03 & 1.98 & $\mathrm{~K}$ \\
\hline 14807.075 & -18.78 & 1.83 & K \\
\hline 14808.057 & -13.83 & 1.96 & $\mathrm{~K}$ \\
\hline 14809.069 & -21.81 & 1.88 & $\mathrm{~K}$ \\
\hline 14810.149 & -15.43 & 1.88 & K \\
\hline 14811.122 & -12.80 & 1.65 & $\mathrm{~K}$ \\
\hline 14847.038 & 3.99 & 1.80 & $\mathrm{~K}$ \\
\hline 14847.048 & 18.53 & 3.83 & $\mathrm{~L}$ \\
\hline 14904.830 & 52.37 & 4.91 & $\mathrm{~L}$ \\
\hline 14929.814 & 59.40 & 1.63 & $\mathrm{~K}$ \\
\hline 14955.878 & 69.74 & 1.60 & $\mathrm{~K}$ \\
\hline 14963.915 & 68.12 & 1.49 & $\mathrm{~K}$ \\
\hline 14978.706 & 55.29 & 4.31 & $\mathrm{~L}$ \\
\hline 14983.770 & 64.81 & 1.59 & $\mathrm{~K}$ \\
\hline 14984.827 & 48.72 & 1.53 & $\mathrm{~K}$ \\
\hline 14987.759 & 63.15 & 1.54 & $\mathrm{~K}$ \\
\hline 14988.750 & 63.85 & 1.60 & $\mathrm{~K}$ \\
\hline 15002.711 & 55.03 & 6.40 & $\mathrm{~L}$ \\
\hline 15014.741 & 57.09 & 1.72 & $\mathrm{~K}$ \\
\hline 15015.750 & 61.18 & 1.58 & $\mathrm{~K}$ \\
\hline 15151.052 & -23.66 & 6.04 & $\mathrm{~L}$ \\
\hline 15164.112 & -21.86 & 1.83 & $\mathrm{~K}$ \\
\hline 15172.150 & -26.29 & 2.05 & $\mathrm{~K}$ \\
\hline 15173.097 & -31.15 & 1.93 & $\mathrm{~K}$ \\
\hline 15189.119 & -40.47 & 1.85 & $\mathrm{~K}$ \\
\hline 15229.007 & -30.27 & 3.76 & $\mathrm{~L}$ \\
\hline 15241.871 & -40.78 & 4.23 & $\mathrm{~L}$ \\
\hline
\end{tabular}

Table 2

(Continued)

\begin{tabular}{lccc}
\hline \hline $\begin{array}{l}\text { JD } \\
-2440000\end{array}$ & $\begin{array}{c}\text { RV } \\
\left(\mathrm{m} \mathrm{s}^{-1}\right)\end{array}$ & $\begin{array}{c}\text { Uncertainty } \\
\left(\mathrm{m} \mathrm{s}^{-1}\right)\end{array}$ & Telescope \\
\hline 15242.872 & -36.26 & 3.75 & $\mathrm{~L}$ \\
15243.855 & -43.99 & 5.27 & $\mathrm{~L}$ \\
15260.941 & -26.13 & 1.65 & $\mathrm{~K}$ \\
15271.797 & -27.09 & 3.77 & $\mathrm{~L}$ \\
15272.789 & -11.58 & 6.00 & $\mathrm{~L}$ \\
15273.750 & -25.81 & 3.66 & $\mathrm{~L}$ \\
15285.854 & -12.17 & 1.75 & $\mathrm{~K}$ \\
15311.810 & -5.48 & 1.43 & $\mathrm{~K}$ \\
15312.791 & 2.81 & 1.56 & $\mathrm{~K}$ \\
\hline
\end{tabular}

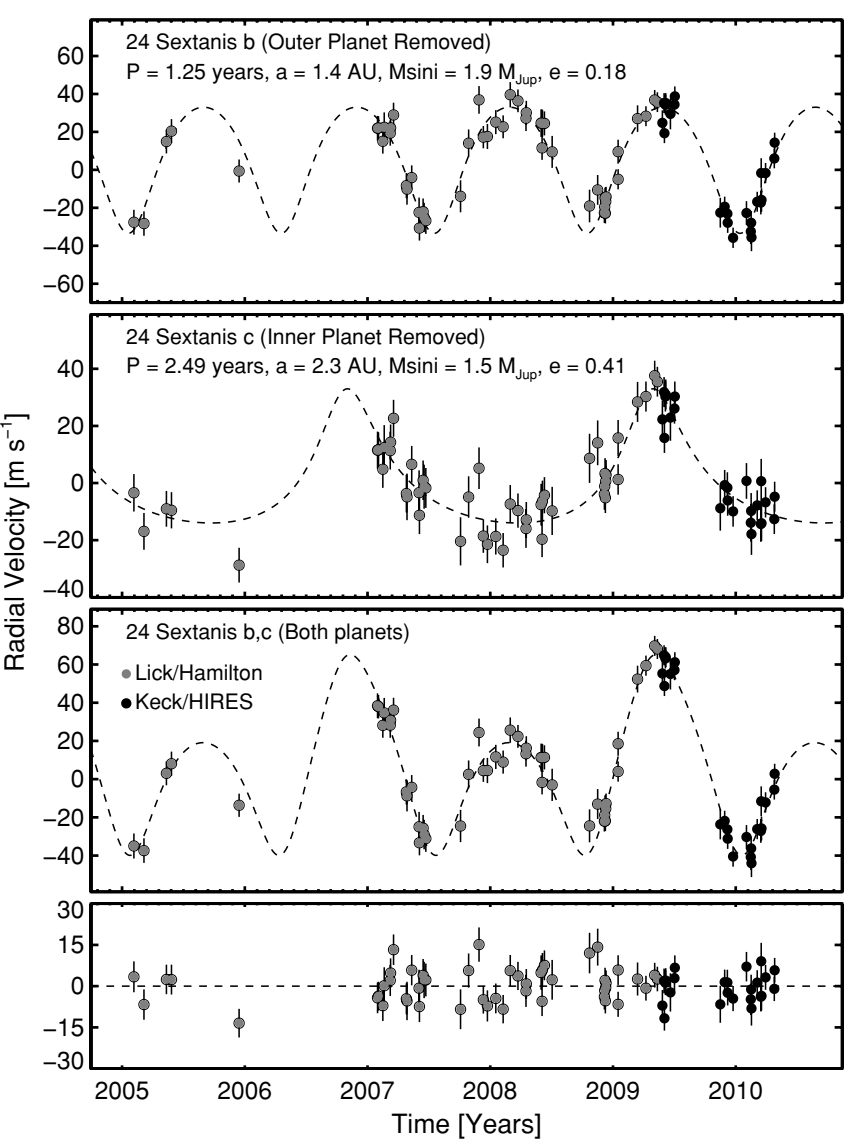

Figure 1. RV time series and two-Keplerian orbital solution for 24 Sex. Top: inner planet with the signal from the outer planet removed. The dashed line shows the best-fitting orbital solution for 24 Sex b. Middle: the outer planet with the inner planet removed. The dashed line shows the best-fitting orbital solution for 24 Sex c. Bottom: the full RV time series, with the two-planet solution shown as a dashed line.

the IDL software suite RVLIN. We estimate the parameter uncertainties using a Markov Chain Monte Carlo (MCMC) algorithm, as described by Bowler et al. (2010).

\subsection{Sextanis}

We obtained initial-epoch observations of 24 Sex at Lick Observatory in 2005 February, and since then we have obtained $50 \mathrm{RV}$ measurements. After noticing time-correlated RV variations, we began additional monitoring at Keck Observatory in 2008 December, where we have obtained 24 additional RV measurements. The RVs from both observatories are listed in Table 2, along with the Julian Dates (JD) of observation and the internal measurement uncertainties (without jitter). Figure 1 
Table 3

Radial Velocities for HD 200964

\begin{tabular}{|c|c|c|c|}
\hline $\begin{array}{l}\text { JD } \\
-2440000\end{array}$ & $\begin{array}{c}\mathrm{RV} \\
\left(\mathrm{m} \mathrm{s}^{-1}\right)\end{array}$ & $\begin{array}{l}\text { Uncertainty } \\
\left(\mathrm{m} \mathrm{s}^{-1}\right)\end{array}$ & Telescope \\
\hline 13213.895 & 11.40 & 4.20 & $\mathrm{~L}$ \\
\hline 13255.775 & 29.55 & 3.72 & $\mathrm{~L}$ \\
\hline 13327.604 & 36.56 & 3.70 & $\mathrm{~L}$ \\
\hline 13576.944 & -20.58 & 3.98 & $\mathrm{~L}$ \\
\hline 13619.810 & -36.28 & 3.71 & $\mathrm{~L}$ \\
\hline 13641.795 & -27.30 & 3.61 & $\mathrm{~L}$ \\
\hline 13669.629 & -18.07 & 3.55 & $\mathrm{~L}$ \\
\hline 13710.605 & -4.99 & 3.77 & $\mathrm{~L}$ \\
\hline 13894.977 & -3.08 & 3.71 & $\mathrm{~L}$ \\
\hline 13895.921 & 6.24 & 3.30 & $\mathrm{~L}$ \\
\hline 13896.963 & 5.08 & 3.47 & $\mathrm{~L}$ \\
\hline 13921.953 & 9.72 & 3.59 & $\mathrm{~L}$ \\
\hline 13959.788 & 7.02 & 3.96 & $\mathrm{~L}$ \\
\hline 14216.976 & -19.74 & 4.09 & $\mathrm{~L}$ \\
\hline 14232.936 & -26.06 & 4.83 & $\mathrm{~L}$ \\
\hline 14244.907 & -27.39 & 5.19 & $\mathrm{~L}$ \\
\hline 14254.960 & -16.97 & 3.70 & $\mathrm{~L}$ \\
\hline 14274.916 & -7.88 & 3.72 & $\mathrm{~L}$ \\
\hline 14288.888 & -3.15 & 3.49 & $\mathrm{~L}$ \\
\hline 14304.877 & 8.18 & 4.89 & $\mathrm{~L}$ \\
\hline 14309.845 & -1.57 & 4.28 & $\mathrm{~L}$ \\
\hline 14336.881 & 16.00 & 4.49 & $\mathrm{~L}$ \\
\hline 14377.773 & 10.58 & 5.74 & $\mathrm{~L}$ \\
\hline 14399.752 & 33.36 & 1.62 & K \\
\hline 14401.749 & 41.83 & 5.67 & $\mathrm{~L}$ \\
\hline 14405.700 & 39.39 & 4.02 & $\mathrm{~L}$ \\
\hline 14427.588 & 35.30 & 4.29 & $\mathrm{~L}$ \\
\hline 14427.757 & 25.14 & 1.18 & K \\
\hline 14429.621 & 49.55 & 4.41 & $\mathrm{~L}$ \\
\hline 14432.653 & 30.85 & 4.89 & $\mathrm{~L}$ \\
\hline 14445.624 & 23.13 & 4.22 & $\mathrm{~L}$ \\
\hline 14536.053 & 20.85 & 5.26 & $\mathrm{~L}$ \\
\hline 14551.019 & 25.15 & 7.90 & $\mathrm{~L}$ \\
\hline 14585.990 & 22.22 & 4.51 & $\mathrm{~L}$ \\
\hline 14603.126 & 14.71 & 1.39 & K \\
\hline 14604.012 & 18.01 & 1.58 & $\mathrm{~K}$ \\
\hline 14612.988 & -7.49 & 6.44 & $\mathrm{~L}$ \\
\hline 14621.965 & 3.21 & 5.14 & $\mathrm{~L}$ \\
\hline 14622.995 & -5.37 & 4.16 & $\mathrm{~L}$ \\
\hline 14634.079 & 10.67 & 1.52 & K \\
\hline 14640.923 & -1.39 & 3.34 & $\mathrm{~L}$ \\
\hline 14650.960 & -13.59 & 3.79 & $\mathrm{~L}$ \\
\hline 14656.902 & 0.00 & 7.81 & $\mathrm{~L}$ \\
\hline 14674.916 & -16.16 & 1.39 & $\mathrm{~K}$ \\
\hline 14675.850 & -16.06 & 4.87 & $\mathrm{~L}$ \\
\hline 14676.879 & -25.35 & 4.49 & $\mathrm{~L}$ \\
\hline 14677.922 & -28.67 & 3.69 & $\mathrm{~L}$ \\
\hline 14683.850 & -22.03 & 4.24 & $\mathrm{~L}$ \\
\hline 14699.804 & -14.57 & 5.28 & $\mathrm{~L}$ \\
\hline 14734.713 & -29.88 & 3.82 & $\mathrm{~L}$ \\
\hline 14737.790 & -29.53 & 5.34 & $\mathrm{~L}$ \\
\hline 14738.751 & -36.28 & 4.54 & $\mathrm{~L}$ \\
\hline 14747.711 & -59.70 & 3.80 & $\mathrm{~L}$ \\
\hline 14766.659 & -48.87 & 3.96 & $\mathrm{~L}$ \\
\hline 14778.803 & -45.21 & 1.61 & $\mathrm{~K}$ \\
\hline 14785.605 & -51.33 & 4.06 & $\mathrm{~L}$ \\
\hline 14790.734 & -47.17 & 1.34 & $\mathrm{~K}$ \\
\hline 14807.789 & -46.94 & 1.60 & $\mathrm{~K}$ \\
\hline 14935.136 & -18.39 & 1.16 & $\mathrm{~K}$ \\
\hline 14941.012 & -18.06 & 6.19 & $\mathrm{~L}$ \\
\hline 14941.989 & -24.98 & 4.38 & $\mathrm{~L}$ \\
\hline 14956.097 & -14.88 & 1.50 & $\mathrm{~K}$ \\
\hline 14964.120 & -5.85 & 1.33 & K \\
\hline 14978.935 & -3.81 & 5.48 & $\mathrm{~L}$ \\
\hline 14984.070 & 6.28 & 1.43 & $\mathrm{~K}$ \\
\hline
\end{tabular}

Table 3

(Continued)

\begin{tabular}{|c|c|c|c|}
\hline $\begin{array}{l}\text { JD } \\
-2440000\end{array}$ & $\begin{array}{c}\mathrm{RV} \\
\left(\mathrm{m} \mathrm{s}^{-1}\right)\end{array}$ & $\begin{array}{l}\text { Uncertainty } \\
\left(\mathrm{m} \mathrm{s}^{-1}\right)\end{array}$ & Telescope \\
\hline 14985.095 & 4.20 & 1.62 & $\mathrm{~K}$ \\
\hline 14986.112 & -1.27 & 1.71 & $\mathrm{~K}$ \\
\hline 14987.129 & 3.06 & 1.39 & $\mathrm{~K}$ \\
\hline 14989.069 & 6.64 & 1.33 & $\mathrm{~K}$ \\
\hline 15002.947 & 32.46 & 5.34 & $\mathrm{~L}$ \\
\hline 15014.972 & 22.36 & 1.54 & $\mathrm{~K}$ \\
\hline 15015.957 & 11.56 & 1.44 & $\mathrm{~K}$ \\
\hline 15027.007 & 22.52 & 1.55 & $\mathrm{~K}$ \\
\hline 15042.973 & 33.72 & 1.69 & $\mathrm{~K}$ \\
\hline 15049.001 & 42.16 & 1.62 & $\mathrm{~K}$ \\
\hline 15060.816 & 45.09 & 4.29 & $\mathrm{~L}$ \\
\hline 15061.819 & 45.31 & 3.69 & $\mathrm{~L}$ \\
\hline 15062.829 & 47.93 & 3.69 & $\mathrm{~L}$ \\
\hline 15075.078 & 45.22 & 1.68 & $\mathrm{~K}$ \\
\hline 15076.067 & 43.59 & 1.75 & $\mathrm{~K}$ \\
\hline 15077.056 & 49.98 & 1.57 & $\mathrm{~K}$ \\
\hline 15082.047 & 45.65 & 1.53 & $\mathrm{~K}$ \\
\hline 15083.053 & 52.10 & 1.63 & $\mathrm{~K}$ \\
\hline 15084.028 & 55.68 & 1.57 & $\mathrm{~K}$ \\
\hline 15085.004 & 46.81 & 1.60 & $\mathrm{~K}$ \\
\hline 15091.772 & 64.86 & 4.56 & $\mathrm{~L}$ \\
\hline 15092.725 & 57.80 & 3.96 & $\mathrm{~L}$ \\
\hline 15106.911 & 57.25 & 1.51 & $\mathrm{~K}$ \\
\hline 15123.798 & 55.22 & 4.81 & $\mathrm{~L}$ \\
\hline 15135.755 & 48.13 & 1.43 & $\mathrm{~K}$ \\
\hline 15148.734 & 61.20 & 4.57 & $\mathrm{~L}$ \\
\hline 15150.620 & 54.74 & 5.97 & $\mathrm{~L}$ \\
\hline 15187.695 & 44.10 & 1.66 & $\mathrm{~K}$ \\
\hline 15188.688 & 41.49 & 1.69 & $\mathrm{~K}$ \\
\hline 15290.146 & 11.79 & 1.39 & $\mathrm{~K}$ \\
\hline 15313.137 & 2.22 & 1.33 & $\mathrm{~K}$ \\
\hline
\end{tabular}

shows the RV time series from both observatories, where the error bars represent the quadrature sum of the internal errors and $5 \mathrm{~m} \mathrm{~s}^{-1}$ of jitter.

Bowler et al. (2010) reported evidence of a two-planet system around 24 Sex, but the data at the time could not provide a unique orbital solution. An additional season of observations has provided stronger constraints on the possible orbits of the two planets. We fitted a model consisting of two non-interacting planets and a $1.54 M_{\odot}$ star orbiting their mutual center of mass. We find that two Keplerians provide an acceptable fit to the data with an rms scatter of $6.8 \mathrm{~m} \mathrm{~s}^{-1}$ and a reduced $\sqrt{\chi_{v}^{2}}=1.14$. The inner planet has a period of $P=455.2 \pm 3.2$ days, velocity semiamplitude $K=$ $33.2 \pm 1.6 \mathrm{~m} \mathrm{~s}^{-1}$, and eccentricity $e=0.184 \pm 0.029$. The outer planet has $P=910 \pm 21$ days, $K=23.5 \pm 2.9 \mathrm{~m} \mathrm{~s}^{-1}$, and $e=0.412 \pm 0.064$. Together with our stellar mass estimate these spectroscopic orbital parameters yield semimajor axes $\left\{a_{b}, a_{c}\right\}=\{1.41,2.22\} \mathrm{AU}$ and minimum planet masses $\left\{M_{b}, M_{c}\right\} \sin i=\{1.6,1.4\} M_{\text {Jup }}$. A more detailed, dynamical analysis presented in Section 6 revises this two-Keplerian solution under the constraint of long-term stability.

\subsection{HD 200964}

We began monitoring HD 200964 at Lick Observatory in 2007 July and have obtained $61 \mathrm{RV}$ measurements. Time-correlated variations in the star's RVs prompted additional monitoring at Keck Observatory where we have obtained 35 measurements since 2007 October. The RVs from both observatories are listed in Table 3, along with the JD of observation and the internal 

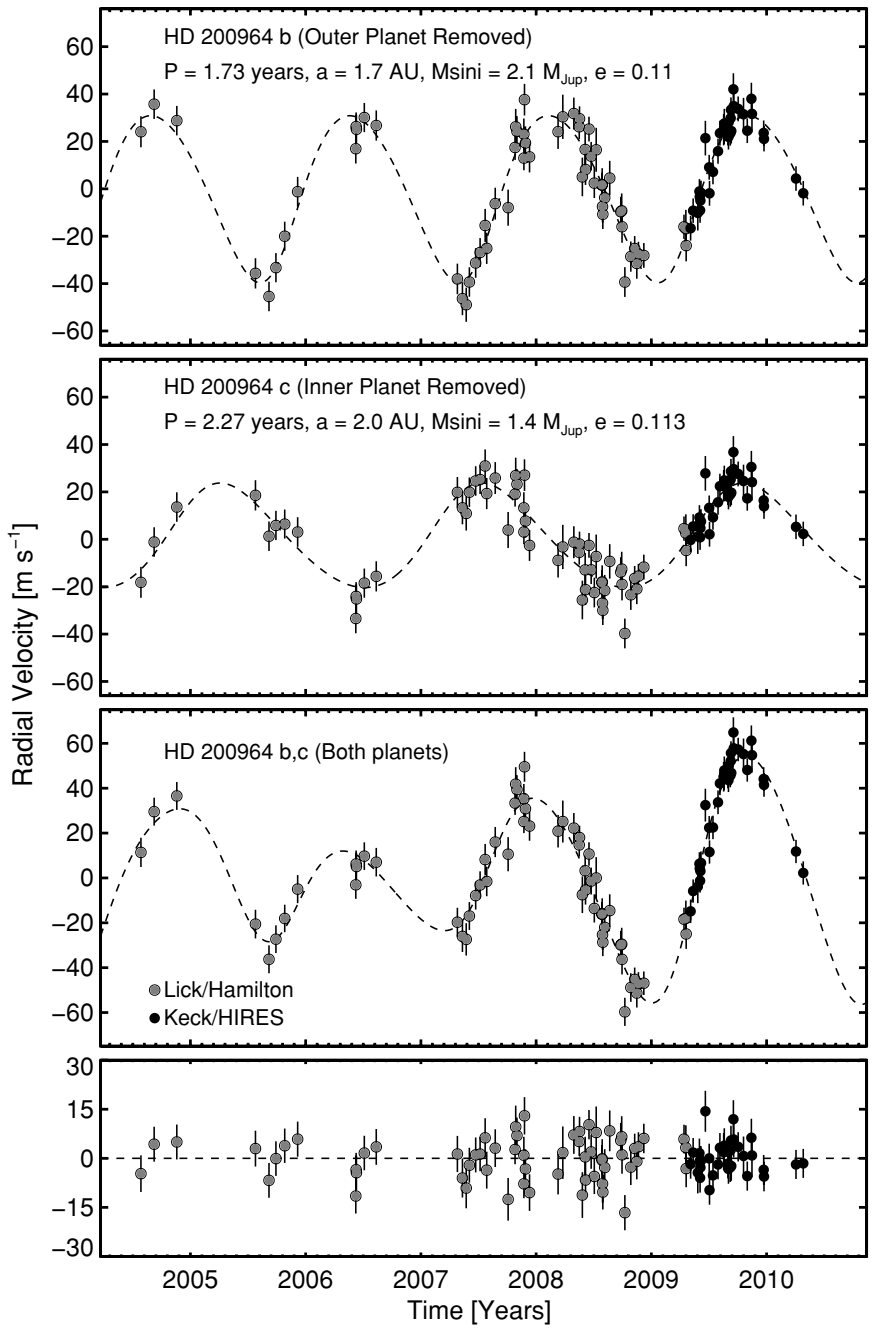

Figure 2. RV time series and two-Keplerian orbital solution for HD 200964. Top: inner planet with the signal from the outer planet removed. The dashed line shows the best-fitting orbital solution for HD 200964 b. Middle: the outer planet with the inner planet removed. The dashed line shows the best-fitting orbital solution for HD 200964 c. Bottom: the full RV time series, with the two-planet solution shown as a dashed line.

measurement uncertainties (without jitter). Figure 2 shows the RV time series from both observatories, where the error bars represent the quadrature sum of the internal errors and $5 \mathrm{~m} \mathrm{~s}^{-1}$ of jitter.

As is the case for 24 Sex, Bowler et al. (2010) reported evidence of a two-planet system around HD 200964, and an additional season of observations has provided stronger constraints on the possible orbits of the planets in the system. We find that a two-Keplerian model provides an acceptable fit to the data with an rms scatter of $6.8 \mathrm{~m} \mathrm{~s}^{-1}$ and a reduced $\sqrt{\chi_{v}^{2}}=1.14$. The inner planet has a period of $P=630.6 \pm 9.3$ days, velocity semiamplitude $K=35.2 \pm 2.7 \mathrm{~m} \mathrm{~s}^{-1}$, and eccentricity $e=0.111 \pm 0.030$. The outer planet has $P=829 \pm 21$ days, $K=22.1 \pm 2.3 \mathrm{~m} \mathrm{~s}^{-1}$, and $e=0.113 \pm 0.076$. Together with our stellar mass estimate these spectroscopic orbital parameters yield semimajor axes $\left\{a_{b}, a_{c}\right\}=\{1.71,2.03\} \mathrm{AU}$ and minimum planet masses $\left\{M_{b}, M_{c}\right\} \sin i=\{1.9,1.3\} M_{\text {Jup }}$. A more in-depth dynamical analysis presented in Section 6 revises this two-Keplerian solution under the constraint of long-term stability.

\section{PHOTOMETRIC MONITORING}

In addition to the radial velocities presented in Section 4 we also acquired photometric measurements of both 24 Sex and HD 200964 with the T12 $0.80 \mathrm{~m}$ Automatic Photometric Telescope (APT) at Fairborn Observatory. The T12 APT and its two-channel photometer measure count rates simultaneously through Strömgren $b$ and $y$ filters. T12 is essentially identical to the T8 $0.80 \mathrm{~m}$ APT described in Henry (1999). Each program star $(P)$ was observed differentially with respect to two nearby comparison stars $(C 1$ and $C 2$ ) (see Table 5). The differential magnitudes $P-C 1, P-C 2$, and $C 2-C 1$ were computed from each set of differential measures. All differential magnitudes with internal standard deviations greater than 0.01 mag were rejected to eliminate observations taken under non-photometric conditions. The surviving observations were corrected for differential extinction with nightly extinction coefficients and transformed to the Strömgren photometric system with yearly mean transformation coefficients. We averaged the $b$ and $y$ measurements of each star into a single $(b+y) / 2$ "passband" (which we designate by in Table 5) to improve the precision of the brightness measurements. Typical precision of a single $(b+y) / 2$ observation, as measured for pairs of constant stars, is $\sim 0.0015-0.0025$ mag on good photometric nights.

Queloz et al. (2001) and Paulson et al. (2004) have demonstrated how rotational modulation in the visibility of star spots on active stars can result in periodic RV variations that mimic the presence of a planetary companion. Thus, the precise APT brightness measurements are valuable for distinguishing between activity-related RV changes and true reflex motion of a star caused by a planet.

Photometric results for 24 Sex and HD 200964 are given in Table 5. Columns 7-9 give the standard deviations of the $P-C 1, P-C 2$, and $C 2-C 1$ differential magnitudes in the $(b+y) / 2$ passbands. All of the standard deviations are small and within the range of measurement precision with the T12 APT. The individual $P-C 1$ and $C 2-C 1$ differential magnitudes for both stars are also plotted in Figure 3 . We also performed periodogram analyses on all the data sets and found no significant periodicity between 0.03 and 100 days that might be the signature of stellar rotation. Our data sets are not sufficiently long to test for variability on the four planetary periods, but we expect any such variability to be very small. The photometric stability of both 24 Sex and HD 200964 and the long-term coherency of the observed RV variations provide strong support for the existence of all four new planets.

\section{DYNAMICAL INTERACTIONS}

Our best-fitting double-Keplerian fits have two Jupitermass planets with periods near the 2:1 commensurability for 24 Sex and near 4:3 for HD 200964. The proximity of the two planets implies strong gravitational interactions, which limits the number of possible orbits to those that allow the two planets to remain stable over the lifetime of the star. To test the long-term stability of the various orbital configurations that are consistent with the data we performed a series of numerical integrations as described in the following sections.

\subsection{Methodology for MCMC Analysis Incorporating n-body Integrations}

We analyze the RV observations using a Bayesian framework following Ford $(2005,2006 a)$. We assume priors that are 
Table 4

Orbital Parameters from Non-interacting Two-planet Solution

\begin{tabular}{lcccc}
\hline \hline Parameter & $24 \mathrm{Sex} \mathrm{b}^{\mathrm{a}}$ & 24 Sex c & HD 200964 b & HD 200964 b \\
\hline Period (days) & $455.2(3.2)$ & $910(21)$ & $630.6(9.3)$ & $829(21)$ \\
$T_{p}$ b (JD) & $2454758(30)$ & $2454941(30)$ & $2454916(30)$ & $2455029(130)$ \\
Eccentricity & $0.184(0.029)$ & $0.412(0.064)$ & $0.111(0.030)$ & $0.113(0.076)$ \\
$K\left(\mathrm{~m} \mathrm{~s}^{-1}\right)$ & $33.2(1.6)$ & $23.5(2.9)$ & $35.2(2.7)$ & $22.1(2.3)$ \\
$\omega(\mathrm{deg})$ & $227(20)$ & $172.0(9)$ & $223(20)$ & $301(50)$ \\
$M_{P} \sin i\left(M_{\text {Jup }}\right)$ & $1.6(0.2)$ & $1.4(0.2)$ & $1.9(0.2)$ & $1.3(0.2)$ \\
$a(\mathrm{AU})$ & $1.41(0.03)$ & $2.22(0.06)$ & $1.71(0.04)$ & $2.03(0.06)$ \\
Lick rms $\left(\mathrm{m} \mathrm{s}^{-1}\right)$ & 7.7 & $\ldots$ & 7.6 & $\ldots$ \\
Keck rms $\left(\mathrm{m} \mathrm{s}^{-1}\right)$ & 4.8 & $\ldots$ & 5.3 & $\ldots$ \\
Jitter $\left(\mathrm{m} \mathrm{s}^{-1}\right)$ & 5.0 & $\ldots$ & 5.0 & $\ldots$ \\
$\sqrt{\chi_{v}^{2}}$ & 1.14 & $\ldots$ & 1.15 & $\ldots$ \\
$N_{\text {obs }}$ Lick & 50 & $\ldots$ & 61 & $\ldots$ \\
$N_{\text {obs Keck }}$ & 24 & $\ldots$ & 35 & $\ldots$ \\
\hline
\end{tabular}

Notes.

${ }^{\mathrm{a}}$ HD 90043.

$\mathrm{b}$ Time of periastron passage.

Table 5

Summary of Photometric Observations From Fairborn Observatory

\begin{tabular}{|c|c|c|c|c|c|c|c|c|c|}
\hline $\begin{array}{c}\text { Program } \\
\text { Star } \\
(1) \\
\end{array}$ & $\begin{array}{c}\text { Comparison } \\
\text { Star } 1 \\
(2) \\
\end{array}$ & $\begin{array}{c}\text { Comparison } \\
\text { Star } 2 \\
(3) \\
\end{array}$ & $\begin{array}{c}\text { Date Range } \\
\text { (HJD - 2400000) } \\
(4) \\
\end{array}$ & $\begin{array}{c}\text { Duration } \\
\text { (days) } \\
(5)\end{array}$ & $\begin{array}{r}N_{\text {obs }} \\
(6) \\
\end{array}$ & $\begin{array}{c}\sigma(P-C 1)_{b y} \\
(\mathrm{mag}) \\
(7)\end{array}$ & $\begin{array}{c}\sigma(P-C 2)_{b y} \\
(\mathrm{mag}) \\
(8)\end{array}$ & $\begin{array}{c}\sigma(C 2-C 1)_{b y} \\
(\mathrm{mag}) \\
(9) \\
\end{array}$ & $\begin{array}{c}\text { Variability } \\
\text { (10) }\end{array}$ \\
\hline $24 \mathrm{Sex}$ & HD 87974 & HD 89734 & $54438-55333$ & 895 & 243 & 0.0022 & 0.0020 & 0.0020 & \\
\hline
\end{tabular}

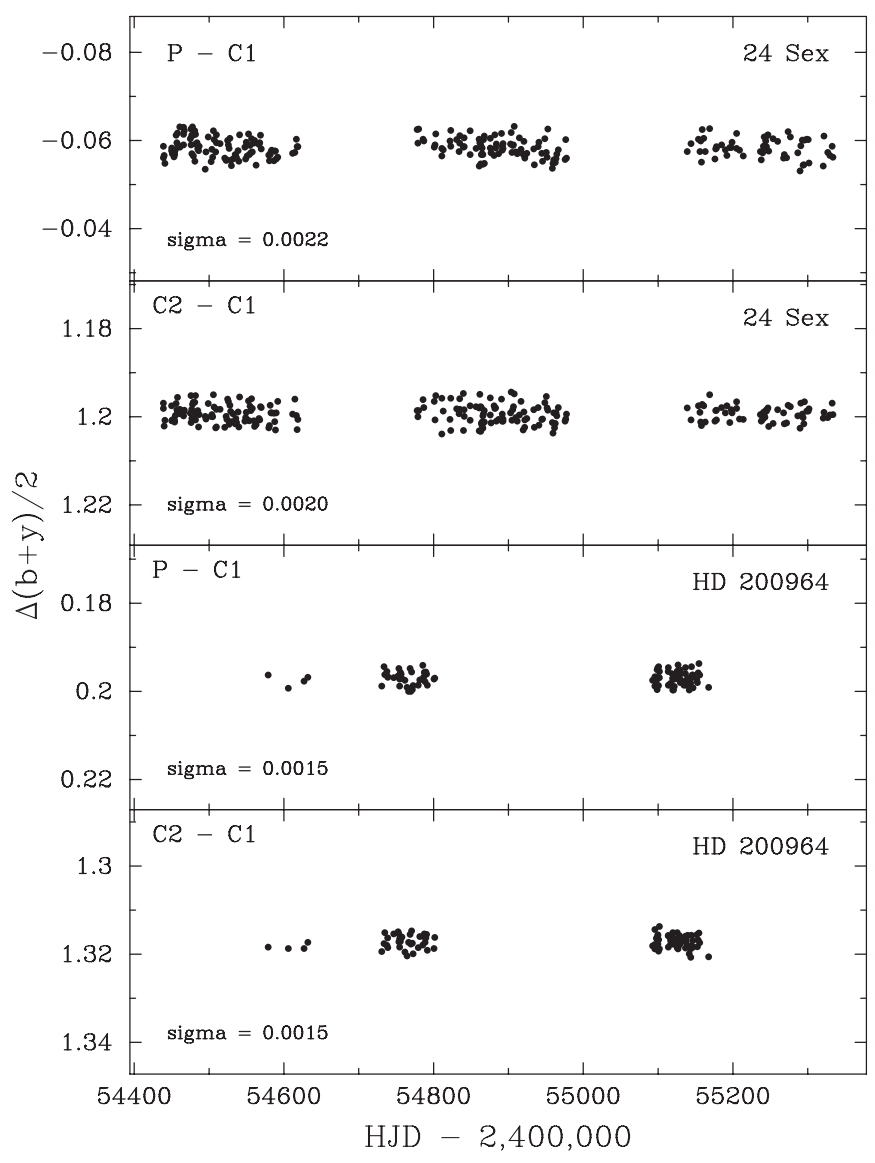

Figure 3. Individual $(b+y) / 2$ differential magnitudes of 24 Sex and HD 200964 from the T12 $0.80 \mathrm{~m}$ APT at Fairborn Observatory. uniform in logarithmic intervals of orbital period, eccentricity, argument of pericenter, mean anomaly at epoch, and the velocity zero point. For the velocity amplitude $(K)$ and stellar jitter $\left(\sigma_{j}\right)$, we adopt a prior of the form $p(x)=\left(x+x_{o}\right)^{-1}\left[\log \left(1+x / x_{o}\right)\right]^{-1}$, with $K_{o}=\sigma_{j, o}=1 \mathrm{~m} \mathrm{~s}^{-1}$. For a discussion of priors, see Ford \& Gregory (2007). The likelihood for RV terms assumes that each RV observation $\left(v_{i}\right)$ is independent and normally distributed about the true RV with a variance of $\sigma_{i}^{2}+\sigma_{j}^{2}$, where $\sigma_{i}$ is the published measurement uncertainty and $\sigma_{j}$ is a jitter parameter that accounts for additional scatter due to stellar variability, instrumental errors, and/or inaccuracies in the model (i.e., neglecting planet-planet interactions or additional, low-amplitude planet signals). It is important to bear in mind that by adding the variance due to jitter to the measurement errors we are making the simplifying assumption that jitter is normally distributed about our model, which is certainly not entirely true as many sources of jitter are systematic rather than purely random.

In our initial phase of analysis, we use an MCMC method based upon Keplerian orbit fitting to calculate a sample from the posterior distribution (Ford 2006a). We calculate multiple Markov chains, each with $\sim 2 \times 10^{8}$ states. We discard the first half of the chains and calculate Gelman-Rubin test statistics for each model parameter and several ancillary variables. We find no indications of non-convergence. Thus, we randomly choose a subsample $(\sim 16,000$ samples $)$ from the posterior distribution for further investigation.

Next, we use this subsample as the basis for a much more computationally demanding analysis that uses fully self-consistent $n$-body integrations to account for planet-planet interactions when modeling the RV observations. We again perform a Bayesian analysis, but replace the standard MCMC algorithm with a Differential Evolution Markov Chain Monte Carlo 
Table 6

Orbital Parameters from Stable, Interacting $n$-Body Two-planet Solution

\begin{tabular}{|c|c|c|c|c|}
\hline Parameter & $24 \operatorname{Sex}^{\mathrm{a}}$ & 24 Sex c & HD $200964 \mathrm{~b}$ & HD $200964 \mathrm{c}$ \\
\hline Period (days) & $452.8\left({ }_{-4.5}^{+2.1}\right)$ & $883.0\left({ }_{-13.8}^{+32.4}\right)$ & $613.8\left(\begin{array}{l}+1.3 \\
-1.4\end{array}\right)$ & $825.0\left(\left(_{-3.1}^{+5.1}\right)\right.$ \\
\hline$T_{p}^{\mathrm{b}}(\mathrm{JD})$ & $2454762\left({ }_{-172.3}^{+67.3}\right)$ & $2454930\left({ }_{-96.5}^{+209.9}\right)$ & $2454900\left({ }_{-89.3}^{+235.5}\right)$ & $2455000\left(\left(_{-54.6}^{+51.1}\right)\right.$ \\
\hline Eccentricity & $0.09\left({ }_{-0.06}^{+0.14}\right)$ & $0.29\left({ }_{-0.09}^{+0.16}\right)$ & $0.04\left({ }_{-0.02}^{+0.04}\right)$ & $0.181\left({ }_{-0.017}^{+0.024}\right)$ \\
\hline$K\left(\mathrm{~m} \mathrm{~s}^{-1}\right)$ & $40.0\left(\begin{array}{c}+4.9 \\
-7.8\end{array}\right)$ & $14.5\left(\begin{array}{l}+7.5 \\
-3.6\end{array}\right)$ & $34.5\left(\begin{array}{l}+2.7 \\
-1.5\end{array}\right)$ & $15.42\left(\begin{array}{c}+2.19 \\
-1.04\end{array}\right)$ \\
\hline$\omega(\operatorname{deg})$ & $9.2\left(\begin{array}{c}+277.9 \\
-165.4\end{array}\right)$ & $220.5\left({ }_{-320.9}^{+182.2}\right)$ & $288.0\left({ }_{-111.9}^{+47.0}\right)$ & $182.6\left(\left(_{-57.1}^{+67.7}\right)\right.$ \\
\hline$M_{P} \sin i\left(M_{\mathrm{Jup}}\right)$ & $1.99\left({ }_{-0.38}^{+0.26}\right)$ & $0.86\left(_{-0.22}^{+0.35}\right)$ & $1.85\left(_{-0.08}^{+0.14}\right)$ & $0.895\left(_{-0.063}^{+0.123}\right)$ \\
\hline$a(\mathrm{AU})$ & $1.333\left({ }_{-0.009}^{+0.004}\right)$ & $2.08\left(\begin{array}{l}+0.05 \\
-0.02\end{array}\right)$ & $1.601\left(\begin{array}{c}0.002 \\
-0.002\end{array}\right)$ & $1.950\left({ }_{-0.005}^{+0.008}\right)$ \\
\hline \multicolumn{5}{|l|}{$\ldots$} \\
\hline Jitter $\left(\mathrm{m} \mathrm{s}^{-1}\right)$ & $9.9\left(\begin{array}{c}+2.9 \\
-1.2\end{array}\right)$ & & $8.23\left({ }_{-0.88}^{+0.38}\right)$ & \\
\hline$\ldots$ & & & & \\
\hline
\end{tabular}

Note. ${ }^{a}$ The figures shown are for the median values obtained from the fitting procedures, while the quoted "error bars" give the $95 \%$ ranges.

(DEMCMC) algorithm (ter Braak 2006; Veras \& Ford 2009, 2010). In the DEMCMC algorithm, each state of the Markov chain is an ensemble of orbital solutions. The candidate transition probability function is based on the orbital parameters in the current ensemble, allowing the DEMCMC algorithm to sample more efficiently from high-dimensional parameter spaces that have strong correlations between model parameters. More details of this DEMCMC algorithm and associated tests of its accuracy will be presented in a forthcoming paper (R. P. Nelson et al. 2011, in preparation)

The priors for model parameters are the same as those of the Keplerian MCMC simulations. The initial conditions of the $n$-body simulations are calculated by converting between Keplerian and Cartesian coordinates. In this paper, we consider only coplanar, edge-on two-planet systems, and hence the planetary masses resulting from this $n$-body analysis (see Table 6) will be minimum masses. We note that relaxing the assumption of coplanarity would (1) be significantly more computationally intensive, and (2) potentially lead to a much greater proportion of unstable systems being generated, as the planetary masses used in the integrations would be increased. Previous experience suggests that inclinations greater than $>45^{\circ}$ frequently lead to highly unstable results, while inclinations $<30^{\circ}$ can often be rather similar to uninclined systems. As such, we emphasize that our results should not be interpreted as giving the precise planetary solutions (masses, period ratios, etc.), but rather as being indicative that stable coplanar solutions exist, and that further detailed investigation is warranted.

For the $n$-body integrations, we use a time-symmetric fourthorder Hermite integrator that has been optimized for planetary systems (Kokubo et al. 1998). We extract the RV of the star (in the barycentric frame) at each of the observation times for comparison to RV data. During the DEMCMC analysis, we also impose the constraint of short-term (100 years) orbital stability. We check whether the planetary semimajor axes remain within a factor of $50 \%$ of their starting value, and that no closeapproaches occur within $0.1 \times$ the semimajor axis during the 100 year $n$-body integration. Any systems failing these tests are rejected as unstable (regardless of the quality of the fit to RV data). Thus, the DEMCMC simulations avoid orbital solutions that are violently unstable. In our DEMCMC simulations, this process is repeated for 10,000 generations, each of which contains 16,000 systems, for a total of $\sim 10^{8} n$-body integrations in each DEMCMC simulation.
Since the DEMCMC simulations only require stability for $\sim 100$ years, the orbital solutions in the final generation may or may not be stable for longer timescales. Since nearly all of these systems are strongly interacting, we take this final generation (16,000 systems) and demand that they also be stable (according to the same criteria above) over the course of a $10^{7}$ year integration, performed using the hybrid BulirschStoer/Symplectic integrator MERCURY (Chambers 1999). Only the orbital solutions which are stable over the course of this long-term $n$-body integration are regarded as being acceptable solutions. More details on the dynamical analysis performed and the results obtained will be presented in a companion paper (M. Payne et al. 2011, in preparation).

\subsection{Numerical Integrations for 24 Sex}

We find that the $n$-body DEMCMC routine results for $24 \mathrm{Sex}$ are concentrated around solutions with $\left\{P_{b}, P_{c}\right\} \approx\{450,900\}$ days, i.e., close to the $2: 1$ resonance, and that they span a significantly smaller range of parameter space than do the double-Keplerian fits. The $n$-body RV fitting aspect of the DEMCMC routine acts to shrink the parameter space, whereas the stability requirement in the routine has the effect of shifting the DEMCMC solutions toward the 2:1 period ratio mark, primarily by favoring lower periods for the inner planet. We illustrate in Figure 4 the difference in the endpoints of the two analyses, showing a scatter plot of the planetary periods at the end of both the Keplerian analysis and the $n$-body analysis.

We have applied the DEMCMC method described in Section 6.1 multiple times using different values for the initial ensemble of orbital solutions. Each time, these simulations reached qualitatively similar results to those of Figure 4, but quantitatively there are signs that the method has not fully converged. Therefore, we do not interpret the results as a precise estimate of the posterior probability distribution. Instead, we use these results to demonstrate that there exist orbital solutions that are both stable and consistent with the Doppler observations. Most importantly, all our simulations indicate that the posterior distribution is concentrated at solutions with a ratio of orbital periods between $\sim 1.9$ and $\sim 2.1$ and very close to the $2: 1$ MMR. Thus, we conclude that the current observations strongly favor orbital solutions in (or at least near) the 2:1 MMR.

Next, we performed long-term stability tests for the 16,000 orbital solutions in the final generation of the $n$-body DEMCMC analysis. We find that $\sim 90 \%$ of the orbital solutions are unstable over the course of $10^{7}$ years of integration. We consider the 

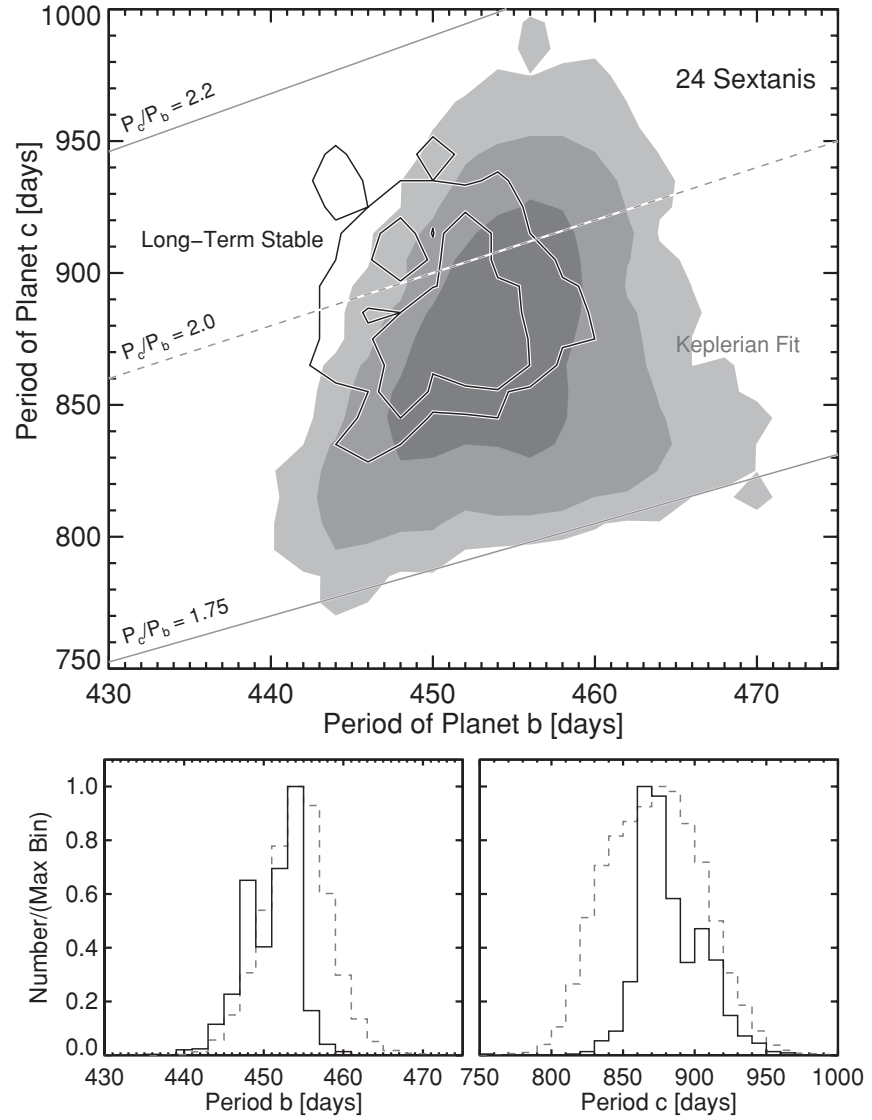

Figure 4. Top: comparison of the endpoints of the double-Keplerian MCMC analysis (shaded contours), and the $n$-body DEMCMC analysis with the longterm-stable constraint (black, solid contours) for 24 Sex. The contours show the $68.2 \%, 80 \%$, and $95 \%$ confidence intervals (inner to outer). To guide the eye, we overlay diagonal lines to indicate $1.75: 1,2: 1$, and 2.2:1 period ratios. The MCMC posterior sample covers a large range of parameter space and is consistent with a 2:1 period ratio. In contrast, the DEMCMC results are limited to a smaller area of parameter space lying on, or close to, the 2:1 period ratio. Bottom: histograms for the same planetary period data from 24 Sex, comparing the results from the Keplerian MCMC analysis (black) and the $n$-body DEMCMC analysis (dark gray). When compared to the basic MCMC results, the DEMCMC results for the inner planet are shifted more toward lower periods, while the outer planet shifts toward higher periods, making the overall period ratio converge more closely toward $2: 1$.

remaining $\sim 1000$ (of the 16,000 orbital solutions) that are stable for $10^{7}$ years to be plausible orbital solutions given both the RV data for $24 \mathrm{Sex}$ and the requirement of long-term dynamical stability.

We show in Figure 4 that the stable systems remain strongly clustered around the 2:1 period commensurability region. Taking all of these long-term-stable systems into account, we find that the data indicate that the inner planet has a period $P_{b}=$ $452.8_{-4.5}^{+2.1}$ days, semimajor axis $a_{b}=1.333_{-0.009}^{+0.004} \mathrm{AU}$, eccentricity $e_{b}=0.09_{-0.06}^{+0.14}$, and mass $M_{b} \sin i=1.99_{-0.38}^{+0.26} M_{\text {Jup }}$, while the outer planet has a period $P_{c}=883.0_{-14}^{+32}$ days, semimajor axis $a_{c}=2.08_{-0.02}^{+0.05} \mathrm{AU}$, eccentricity $e_{c}=0.29_{-0.09}^{+0.16}$, and mass $M_{c} \sin i=0.86_{-0.22}^{+0.35} M_{\text {Jup }}$. We detail all the fitted parameters from this analysis in Table 6.

When we examine specific systems in detail, we find that the pericenter of the outer planet overlaps the location of the pericenter of the inner planet, meaning that the planets therefore experience detectable gravitational interactions. Individual systems typically undergo short-term eccentricity and semimajor axis oscillations with amplitudes that can be at least as large as the spread in median values quoted in Table 6. As an example, one stable system was found to exhibit planetary eccentricity oscillations with a period $\sim 800$ years and amplitude $0.02<e_{b}<0.20$ and $0.15<e_{c}<0.36$, while the semimajor axes oscillated with a period $\sim 25$ years and amplitudes $1.330<a_{b}<1.338$ AU and $2.05<a_{c}<2.12$ AU.

We note that the PDFs from the DEMCMC analysis are bimodal. To investigate the cause of the bimodality, we performed a similar $n$-body+DEMCMC analysis without the requirement of short-term stability. The resulting PDFs have a single broader mode consistent with the output of the Keplerian MCMC analysis. We conclude that the bimodal nature of the PDF from the dynamical analysis is most likely the result of demanding short-term stability.

The overlap of the pericenters suggests that an MMR is needed to stabilize the system over long timescales by preventing close encounters. However, an analysis of the resonant angles $\theta_{2,1}=2 \lambda_{c}-1 \lambda_{b}-1 \varpi_{2,1}$ (where $\lambda$ is the mean longitude and $\varpi$ is the longitude of pericenter) suggests that, for some of the long-term-stable systems, the planets circulate, rather than librate, i.e., the systems are observed to have angular ranges for $\theta_{2,1} \sim 360^{\circ}$. We therefore caution that the current state of the observations and dynamical analysis cannot confirm whether the system is truly in a resonant state, and hence further work will be required. A more detailed investigation (M. Payne et al. 2011 , in preparation) will probe the nature of these dynamical interactions in 24 Sex and HD200964 in greater detail.

Finally, we note that, as discussed in Section 6.1, jitter is allowed to vary during the DEMCMC procedure. As such we find a best-fit value for the jitter in the same manner as we do for the various planetary orbital parameters. From the 24 Sex analysis, we find a large jitter value of $9.9_{-1.2}^{+2.9} \mathrm{~m} \mathrm{~s}^{-1}$. This is substantially larger than the value of $5 \mathrm{~m} \mathrm{~s}^{-1}$ assumed in Section 4, indicating that 24 Sex has intrinsic RV variability at the high end of the observed distribution, or that there are other unmodeled sources of variability such as an additional planet in the system. However, a periodogram of the residuals shows no significant power above the noise. Using the approach of Bowler et al. (2010), we can rule out additional companions with masses greater than $0.3 M_{\text {Jup }}$ out to $1 \mathrm{AU}$ and "hot" planets with masses $M_{P} \sin i<0.1 M_{\text {Jup }}$ within 0.1 AU. Additional monitoring at Keck and a more in-depth dynamical analysis will help clarify the situation.

\subsection{Numerical Integrations for HD 200964}

The results of our $n$-body DEMCMC simulations for HD 200964 are again more tightly confined than the results of the double-Keplerian analysis. As an example of this we illustrate in Figure 5 the difference in the endpoints of the two analyses, showing a scatter plot of the planetary periods at the end of both the Keplerian analysis and the $n$-body analysis, as well as histograms for the same data. Again, we note that the shift to an $n$-body analysis acts to concentrate the preferred region into a smaller area, while the stability requirements act to shift the favored parameters closer toward the 4:3 period commensurability.

As for the case of 24 Sex, we have applied our DEMCMC method multiple times using numerous randomized initial conditions. Again, we find qualitatively similar results from all sets of the DEMCMC analysis, but with some indication that the results have not truly converged. As such, we interpret these results as demonstrative that stable systems exist which 

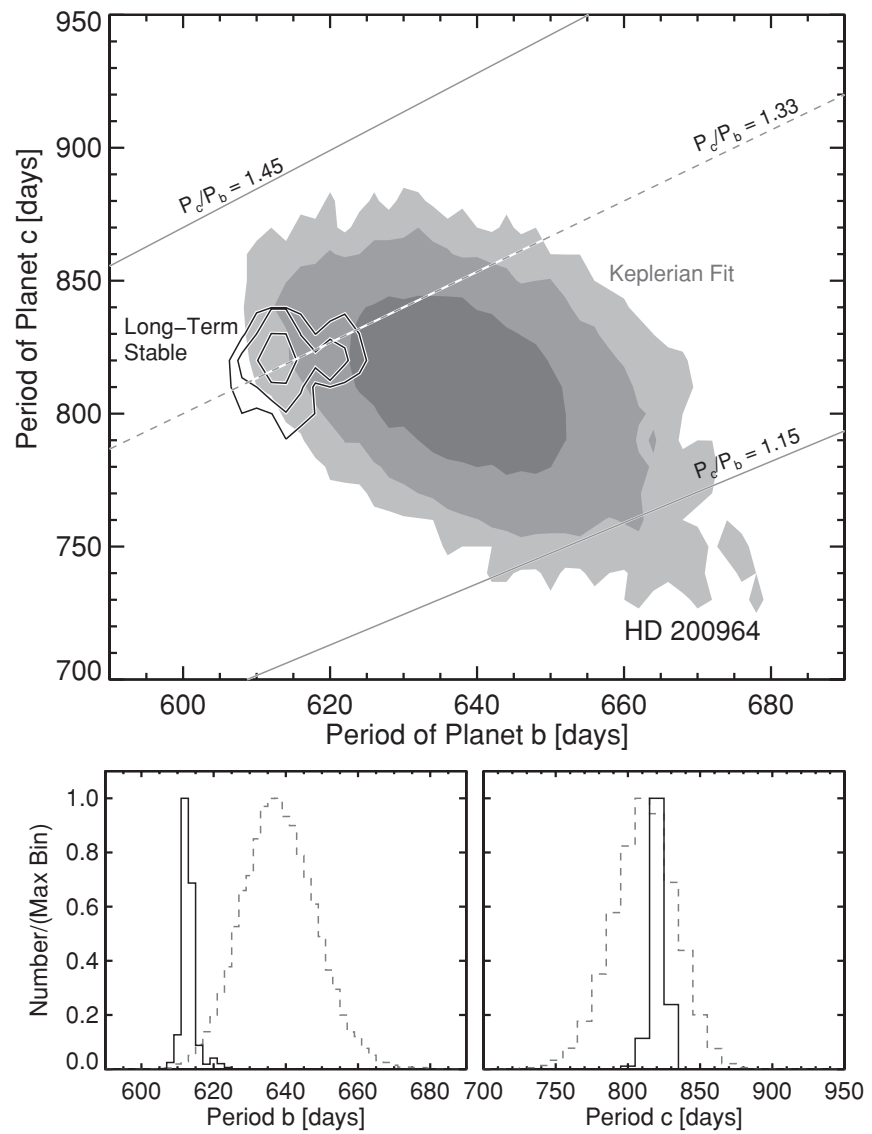

Figure 5. Top: comparison of the endpoints of the double-Keplerian MCMC analysis (shaded contours), and the $n$-body DEMCMC analysis with the longterm-stable constraint (black, solid contours) for 24 Sex. The contours show the $68.2 \%, 80 \%$, and $95 \%$ confidence intervals (inner to outer). To guide the eye, we overlay diagonal lines to indicate $1.15: 1,4: 3$, and $1.45: 1$ period ratios. The MCMC posterior sample covers a large range of parameter space and is consistent with a 4:3 period ratio. In contrast, the DEMCMC results are limited to a smaller area of parameter space straddling the $4: 3$ period ratio. Bottom: histograms for the two planetary periods in the HD 200964 system, comparing the results from the Keplerian MCMC analysis (black) and $n$-body DEMCMC analysis (dark gray). When compared to the basic MCMC results, the DEMCMC analysis results in a decrease in the fitted period of the inner planet, making the overall period ratio converge more closely toward 4:3.

can fit the RV data, and moreover, the extremely narrow range of period ratios favored by the analysis $(\sim 1.32$ to $\sim 1.36)$ shows that the current observations strongly favor orbital solutions in or close to the 4:3 MMR.

Next, we test the long-term orbital stability of the orbital solutions identified by the DEMCMC analysis. For HD 200964 , $>90 \%$ of the systems are clearly unstable during a $10^{7}$ year integration (i.e., they experience a collision or a change in semimajor axes of more than $50 \%$.) We find that $\sim 1000$ of the 16,000 simulations remain stable for $10^{7}$ years. We interpret these as plausible orbital solutions consistent with the RV observations of HD 200964.

As illustrated in Figure 5, the stable systems occupy a region of parameter space corresponding to a region of parameter space near the 4:3 period ratio. Taking only these long-term-stable systems into account, we find that the inner planet has a period $P_{b}=613.8_{-1.4}^{+1.3}$ days, semimajor axis $a_{b}=1.601_{-0.002}^{0.002} \mathrm{AU}$, eccentricity $e_{b}=0.04_{-0.02}^{+0.04}$, and mass $M_{b} \sin i=1.85_{-0.08}^{+0.14} M_{\mathrm{Jup}}$, while the outer planet has a period $P_{c}=825.0_{-3.1}^{+5.1}$ days, semimajor axis $a_{c}=1.95_{-0.005}^{+0.008} \mathrm{AU}$, eccentricity $e_{c}=0.181_{-0.017}^{+0.024}$, and mass $M_{c} \sin i=0.90_{-0.06}^{+0.12} M_{\mathrm{Jup}}$. We detail all the fitted parameters from this analysis in Table 6.

We find that most of the stable planetary orbits overlap, producing strongly interacting systems, resulting in significant oscillations in the semimajor axes and the eccentricities of both planets. An example stable solution exhibits observable eccentricity oscillations $\left(0.03<e_{b}<0.1\right.$ and $\left.0.13<e_{c}<0.18\right)$ on an approximately 250 year timescale and semimajor axis oscillations $\left(1.57<a_{b}<1.58 \mathrm{AU}\right.$ and $\left.1.9<a_{c}<1.93 \mathrm{AU}\right)$ on an approximately 60 year timescale. As in the 24 Sex system, some of the long-term-stable systems examined here for HD 200964 seem to be circulating rather librating and we are conducting a more detailed examination to ascertain the fraction of system solutions which actually librate. We find a jitter value of $8.23_{-0.88}^{+0.38} \mathrm{~m} \mathrm{~s}^{-1}$, which is larger than the empirical jitter estimate of Johnson et al. (2010).

\section{SUMMARY AND DISCUSSION}

Our RV measurements of the intermediate-mass subgiants 24 Sex and HD $200964\left(M_{\star}=1.54\right.$ and $1.44 M_{\odot}$, respectively) reveal the presence of a pair of giant planets around each star. Our orbital analysis indicates that most, if not all, of the dynamically stable solutions include crossing orbits, suggesting that each system is locked in an MMR that prevents close encounters and provides long-term stability. The planets in the 24 Sex system likely have a period ratio near $2: 1$, while the HD 200964 system is even more tightly packed with a period ratio close to $4: 3$.

In both the 24 Sex and HD 200964 systems, the planets reside well within the so-called snow line, beyond which volatiles in the protoplanetary disk can condense to provide the raw materials for protoplanetary core growth. For a premain-sequence, $1.5 M_{\odot}$ star the snow line is located beyond $\approx 3$ $\mathrm{AU}$ according to the estimates of Kennedy \& Kenyon (2008) for realistic disk models including irradiation and accretional heating. It is therefore likely that the planets around 24 Sex and HD 200964 formed at larger semimajor axes and subsequently migrated inward to their current locations at $a<2.5 \mathrm{AU}$ (see Papaloizou \& Terquem 2006 for a review of migration theory).

In addition to their current locations inside the ice line, the resonant configurations of the planetary systems around 24 Sex and HD 200964 provide additional evidence of inward migration. Unless the planets around 24 Sex and HD 200964 formed with period ratios close to their current values, the pairs of planets in each system must have moved from their birthplaces into their current tight configurations via a mechanism such as planet-planet scattering (e.g., Adams \& Laughlin 2003) or through disk interactions (e.g., Kley 2000). Planets migrating through disk interactions may do so at convergent rates until they became trapped in an MMR, with the strong 2:1 resonance being the most common endpoint of such differential migration (Kley 2000; Nelson \& Papaloizou 2002). Indeed, the observed rarity of planets discovered with period ratios smaller than 2:1 accords well with the dynamical simulations of Lee et al. (2009). In their simulation they considered the formation of two giant planets in a protoplanetary disk with initial period ratios just outside of 2:1 and final orbital configurations determined by the initial conditions and details of the planet-planet-disk interactions. From their ensemble of simulated planetary systems they found that only $3 \%$ attain period ratios closer than $2: 1$, and none ended in stable configurations closer than 3:2. 
The ability of planets to reach a resonance deeper than $3: 2$ is dependent upon a number of factors including the initial separation of the planets, disk viscosity, planet masses, remaining disk mass, and size of the gaps opened by the planets (Malhotra 1993; Haghighipour 1999; Bryden et al. 2000; Snellgrove et al. 2001). One key variable is the convergent migration rate, which if fast enough can carry the planets past the deep 2:1 MMR into closer commensurabilities. The 24 Sex system is near the 2:1 resonance, which may be a reflection of a slower migration process compared to that which led to the extremely tight 4:3 configuration in the otherwise very similar HD 200964 system.

Papaloizou \& Szuszkiewicz (2010) explored rapid migration scenarios leading to the attainment of high-order MMRs by low-mass planets migrating within a gas disk. For the terrestrial planets they considered, MMRs with degrees as high as 8:7 and 11:10 were achieved for migration timescales of order $10^{3}$ years. However, to test whether such conditions can lead to highorder stable MMRs for the planets in the HD 200964 system, and the 2:1 MMR seen in the 24 Sex system, hydrodynamic considerations need to be incorporated into the models. Rein et al. (2010) investigated the formation and evolution of the gas giants orbiting HD 45364 and found plausible models for the attainment of the 3:2 MMR observed in that system. Simulations of this nature are beyond the scope of the present work and will be presented in a future contribution (M. Payne et al. 2011, in preparation). For now, it is clear that, just like the resonant planetary systems discovered before them, the 24 Sex and HD 200964 systems pose interesting challenges to theories of planet formation and orbital evolution.

We thank the many observers who contributed to the observations reported here. We gratefully acknowledge the efforts and dedication of the Keck Observatory staff, especially Grant Hill, Scott Dahm, and Hien Tran for their support of HIRES and Greg Wirth for support of remote observing. We are also grateful to the time assignment committees of NASA, NOAO, Caltech, and the University of California for their generous allocations of observing time. M.J.P. thanks Stan Peale for his insightful comments regarding the likely libration states of the systems. A.W.H. gratefully acknowledges support from a Townes Post-doctoral Fellowship at the U. C. Berkeley Space Sciences Laboratory. E.B.F. and M.J.P. were supported by NASA Origins of Solar Systems grant NNX09AB35G. G.W.M. acknowledges NASA grant NNX06AH52G. G.W.H acknowledges support from NASA, NSF, Tennessee State University, and the State of Tennessee through its Centers of Excellence program. Finally, the authors wish to extend special thanks to those of Hawaiian ancestry on whose sacred mountain of Mauna Kea we are privileged to be guests. Without their generous hospitality, the Keck observations presented herein would not have been possible.

\section{REFERENCES}

Adams, F. C., \& Laughlin, G. 2003, Icarus, 163, 290

Bakos, G. Á., et al. 2009, ApJ, 707, 446

Batygin, K., Bodenheimer, P., \& Laughlin, G. 2009, ApJ, 704, L49
Bowler, B. P., et al. 2010, ApJ, 709, 396

Bryden, G., et al. 2000, ApJ, 540, 1091

Butler, R. P., et al. 1996, PASP, 108, 500

Butler, R. P., et al. 1999, ApJ, 526, 916

Cameron, A. C., et al. 2007, MNRAS, 375, 951

Chambers, J. E. 1999, MNRAS, 304, 793

Correia, A. C. M., et al. 2010, A\&A, 511, A21

Fischer, D. A., \& Valenti, J. 2005, ApJ, 622, 1102

Ford, E. B. 2005, AJ, 129, 1706

Ford, E. B. 2006a, ApJ, 642, 505

Ford, E. B. 2006b, in ASP Conf. Ser. 352, New Horizons in Astronomy: Frank N. Bash Symposium, ed. S. J. Kannappan et al. (San Francisco, CA: ASP), 15

Ford, E. B., \& Gregory, P. C. 2007, in ASP Conf. Ser. 371, Statistical Challenges in Modern Astronomy IV, ed. G. Jogesh Babu \& E. D. Feigelson (San Francisco, CA: ASP), 189

Ford, E. B., Lystad, V., \& Rasio, F. A. 2005, Nature, 434, 873

Gaudi, B. S., et al. 2008, Science, 319, 927

Gould, A., et al. 2010, ApJ, 720, 1073

Haghighipour, N. 1999, MNRAS, 304, 185

Henry, G. W. 1999, PASP, 111, 845

Johnson, J. A., et al. 2006a, ApJ, 652, 1724

Johnson, J. A., et al. 2006b, ApJ, 647, 600

Johnson, J. A., et al. 2007, ApJ, 665, 785

Johnson, J. A., et al. 2008, ApJ, 675, 784

Johnson, J. A., et al. 2010, PASP, 122, 701

Kalas, P., et al. 2008, Science, 322, 1345

Kennedy, G. M., \& Kenyon, S. J. 2008, ApJ, 673, 502

Kley, W. 2000, MNRAS, 313, L47

Kokubo, E., Yoshinaga, K., \& Makino, J. 1998, MNRAS, 297, 1067

Laughlin, G., et al. 2005, ApJ, 622, 1182

Lee, A. T., Thommes, E. W., \& Rasio, F. A. 2009, ApJ, 691, 1684

Lee, M. H. 2004, ApJ, 611, 517

Malhotra, R. 1993, Icarus, 106, 264

Malhotra, R. 2002, ApJ, 575, L33

Marcy, G. W., \& Butler, R. P. 1992, PASP, 104, 270

Marcy, G. W., et al. 2001, ApJ, 556, 296

Mardling, R. A. 2010, MNRAS, 407, 1048

Marois, C., et al. 2008, Science, 322, 1348

Nelson, R. P., \& Papaloizou, J. C. B. 2002, MNRAS, 333, L26

Noyes, R. W., et al. 1984, ApJ, 279, 763

Papaloizou, J. C. B., \& Szuszkiewicz, E. 2010, 42, 333

Papaloizou, J. C. B., \& Terquem, C. 2006, Rep. Prog. Phys., 69, 119

Paulson, D. B., et al. 2004, AJ, 127, 1644

Peek, K. M. G., et al. 2009, PASP, 121, 613

Queloz, D., et al. 2001, A\&A, 379, 279

Rein, H., Papaloizou, J. C. B., \& Kley, W. 2010, A\&A, 510, A4

Rivera, E. J., et al. 2005, ApJ, 634, 625

Saar, S. H., Butler, R. P., \& Marcy, G. W. 1998, ApJ, 498, L153

Snellgrove, M. D., Papaloizou, J. C. B., \& Nelson, R. P. 2001, A\&A, 374, 1092

ter Braak, C. F. T. 2006, Stat. Comput., 16, 239

Valenti, J. A., Butler, R. P., \& Marcy, G. W. 1995, PASP, 107, 966

Valenti, J. A., \& Fischer, D. A. 2005, ApJS, 159, 141

Valenti, J. A., \& Piskunov, N. 1996, A\&AS, 118, 595

Valenti, J. A., et al. 2009, ApJ, 702, 989

van Leeuwen, F. 2007, A\&A, 474, 653

VandenBerg, D. A., \& Clem, J. L. 2003, AJ, 126, 778

Veras, D., \& Ford, E. B. 2009, ApJ, 690, L1

Veras, D., \& Ford, E. B. 2010, ApJ, 715, 803

Vogt, S. S. 1987, PASP, 99, 1214

Vogt, S. S., et al. 1994, Proc. SPIE, 2198, 362

Winn, J. N., et al. 2010, ApJ, 718, 575

Wright, J. T. 2005, PASP, 117, 657

Wright, J. T., \& Howard, A. W. 2009, ApJS, 182, 205

Wright, J. T., et al. 2004, ApJS, 152, 261

Wright, J. T., et al. 2009, ApJ, 693, 1084

Wu, Y., \& Goldreich, P. 2002, ApJ, 564, 1024

Yi, S. K., Demarque, P., \& Kim, Y.-C. 2004, Ap\&SS, 291, 261 